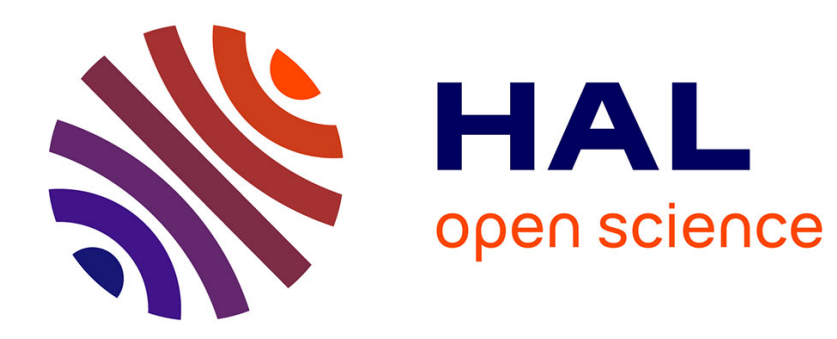

\title{
Rénovation urbaine. Une mixité très peu sociale
} Thomas Kirszbaum

\section{To cite this version:}

Thomas Kirszbaum. Rénovation urbaine. Une mixité très peu sociale. Revue Projet, 2008, pp.30-37. halshs-01100111

\section{HAL Id: halshs-01100111 https://shs.hal.science/halshs-01100111}

Submitted on 5 Jan 2015

HAL is a multi-disciplinary open access archive for the deposit and dissemination of scientific research documents, whether they are published or not. The documents may come from teaching and research institutions in France or abroad, or from public or private research centers.
L'archive ouverte pluridisciplinaire HAL, est destinée au dépôt et à la diffusion de documents scientifiques de niveau recherche, publiés ou non, émanant des établissements d'enseignement et de recherche français ou étrangers, des laboratoires publics ou privés. 
énovation urbaine Une mixité très peu sociale

\author{
Thomas Kirszbaum
}

Une recherche menée dans neuf communes d'lle de France : les politiques locales de mixité facilitent-elles la mobilité des minorités ethniques ou sont-elles sources de discrimination?

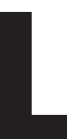

e modèle français d'action publique se prétend aveugle à toute distinction ethnique et raciale. Il est pourtant loin d'être absolument Thermétique, dans la pratique, à la catégorisation des populations sur de tels fondements. Les études s'accumulent depuis une dizaine d'années pour décrire des formes d'ethnicisation occulte de l'action publique, qu'il s'agisse de l'école, de l'armée, des services de l'emploi, de santé, de police, de justice ou des services publics dans les quartiers défavorisés ${ }^{1}$. Ces recherches soulignent les effets discriminatoires des classements que manipulent, souvent à leur insu, les agents des services publics. D'autres auteurs avancent, en revanche, l'hypothèse d'une discrimination positive «à la française » qui pourrait se passer des caté-

\footnotetext{
1. Françoise Lorcerie (dir.), L'école et le défi ethnique. Éducation et intégration, ESF, 2003. C. Bertossi, Catherine Wihtol de Wenden, Les couleurs du drapeau: l'armée française face aux discriminations, R. Laffont, 2007. O. Noël, «La face cachée de l'intégration : les discriminations institutionnelles à l'embauche ", VEI-enjeux, n 121, juin 2000. E. Carde, «Les discriminations selon l'origine dans l'accès aux soins ", Santé publique, ${ }^{\circ}$ 2, mars-avril 2007. Sophie Body-Gendrot, C. Wihtol de Wenden, Police et discriminations. Le tabou français, éd. de l'Atelier, éd. Ouvrières, 2003. F. Jobard, S. Névanen, "La couleur du jugement: discriminations dans les décisions judiciaires ", Revue française de sociologie, vol. 48, $\mathrm{n}^{\circ}$ 2, 2007. Annie Maguer, "Catégorisations institutionnelles et spontanées dans les services publics ", Mouvements, n 4, mai-juillet 1999.
}

Thomas Kirszbaum est consultant (REPS), chercheur associé au GEPECS (Université Paris V). 
gories ethno-raciales pour leur substituer d'autres critères, sociaux ou territoriaux, afin de promouvoir la "diversité $»^{2}$.

Le logement social fournit un bon exemple de cette ambivalence du modèle républicain français. Le principe de mixité dite "sociale " pourrait inspirer une discrimination positive si la production de logements sociaux et les filières d'attribution étaient mobilisées pour augmenter les chances d'accès des minorités ethniques aux territoires qui leur sont fermés. Accolé au terme "mixité ", l'épithète "sociale " permettrait de contourner les effets stigmatisants d'une classification explicitement ethno-raciale. Telle est la lecture qui pouvait être faite des dispositifs de lutte contre la ségrégation urbaine adoptés au début des années $90^{3}$. Cette approche ne s'est pourtant jamais concrétisée. Nulle part la mixité sociale a motivé l'ouverture de territoires aux minorités ethniques, à l'instar des stratégies (prudentes) de promotion de la « diversité » dans les entreprises ou l'audiovisuel.

\section{" Déconcentration "}

Une récente circulaire gouvernementale sur « l'égalité des chances dans l'accès au logement " semble marquer un tournant. S'affranchissant de la pudeur habituelle des pouvoirs publics qui définissaient jusque-là les publics "prioritaires" par de seuls critères socio-économiques, cette circulaire demande que les populations "notamment étrangères" se voient proposer des occasions de logement dans les segments du parc social où elles sont sous-représentées, c'est-à-dire ailleurs que dans les zones urbaines sensibles (Zus) ${ }^{4}$. Mais, outre le caractère très restrictif du critère de la nationalité, l'action positive esquissée en direction des "étrangers " est contrebalancée par une stigmatisation récurrente de

2. Voir G. Calvès, "Affirmative Action in french Law", Revue Tocqueville, vol 19 n², 1998; et D. Sabbagh, "Une convergence problématique : les stratégies de légitimation de la 'discrimination positive' dans l'enseignement supérieur aux Etats-Unis et en France », Politix, n 1, 2006.

3. On pense à la loi Besson sur la mise en œuvre du droit au logement et à la loi d'orientation pour la ville dite loi anti-ghettos. Voir T. Kirszbaum et P. Simon, "Les discriminations raciales et ethniques dans l'accès au logement social ", Notes du GELD, n³, 2001.

4. Circulaire du ministre de l'Immigration, de l'intégration, de l'identité nationale et du développement solidaire, et du ministre du Logement et de la ville du 18 juin 2008 relative à " un plan d'actions pour favoriser l'égalité des chances dans l'accès au logement pour les populations, en particulier étrangères, cumulant difficultés économiques et sociales ». 
leurs « concentrations territoriales » : le mot " concentration » apparaît quelque 25 fois dans la circulaire et son annexe!

Ce texte souligne en particulier que «sans action sur la composition de la population, le succès à terme d'actions aussi fondamentales que les opérations de rénovation urbaine peut être fortement compromis ». Les premières évaluations des relogements effectués à la suite des démolitions engagées dans les quartiers dits sensibles, au nom de la rénovation urbaine et de la mixité sociale, montrent en effet que les habitants sont massivement relogés dans des quartiers de même nature ${ }^{5}$ La circulaire entend ainsi remédier à une incohérence majeure de la stratégie de déconcentration spatiale des minorités ethniques, jusqu'ici fondée sur un raisonnement tacite : celui d'une complémentarité entre l'éradication des " ghettos " par la démolition de logements sociaux, d'un côté, et le développement d'une offre sociale dans les villes déficitaires ${ }^{6}$, de l'autre, afin de créer les conditions d'un transfert territorial de populations.

En attendant que se concrétise une action positive facilitant la mobilité résidentielle des minorités ethniques vers les segments "protégés " $\mathrm{du}$ parc social ou les villes qui n'ont pas encore atteint le fameux seuil de $20 \%$ de logements sociaux, la mixité sociale reste ce qu'elle n'a cessé d'être depuis la fin des années 90 : un argument potentiellement discriminatoire (au sens négatif du terme) pour modifier le peuplement des " cités " - et seulement de celles-ci - en agissant sur leur composition résidentielle. À défaut d'avoir inspiré une formule de discrimination positive centrée sur la mobilité résidentielle des minorités, la mixité sociale peut en effet restreindre leurs opportunités résidentielles dans les quartiers où les acteurs des politiques de l'habitat estiment que ces populations sont en surnombre.

\section{Les êlus face à la mixité}

Limitée aux seules Zus, la rénovation urbaine est aujourd'hui le principal instrument de transformation volontariste des équilibres socio-

5. C. Lelévrier, Mobilités et trajectoires résidentielles des ménages relogés lors d'opérations de renouvellement urbain. Synthèse de travaux menés entre 2004 et 2007, PUCA, DIV, DREIF, mars 2008.

6. Au sens de la loi d'orientation pour la ville de 1991, puis de la loi SRU de 2000. 
logiques dans la ville. Jusqu'à quel point cette politique reflète-t-elle le processus d'ethnicisation du modèle français d'action publique? En quoi les acteurs locaux de la rénovation urbaine cherchent-ils à transformer la composition ethno-raciale des quartiers en s'abritant derrière le vocabulaire de la mixité sociale? C'est l'une des questions que nous avons cherché à élucider au cours d'une recherche menée dans neuf communes franciliennes, représentatives d'une variété de contextes politiques et urbains ${ }^{7}$.

Pour une moitié des 39 élus interrogés, la concentration spatiale de minorités ethniques est un problème en soi auquel l'action publique se doit de répondre. Dans cet esprit, la mixité suppose non seulement le rassemblement d'une diversité de groupes sociaux sur un territoire donné, mais aussi une diversité d'origines ethno-raciales. La nationalité française n'étant pas un critère suffisant, c'est la présence en nombre significatif de "Français de souche " qui, pour ces élus, paraît constitutif d'une situation de mixité sociale. Ceux qui rejettent cette lecture au profit d'une analyse strictement socio-économique de la mixité sont très minoritaires dans notre échantillon. Un dernier groupe est plus mitigé, hésitant entre une analyse qui accorde la primauté aux critères socio-économiques ou aux critères ethno-raciaux et culturels de la mixité ${ }^{8}$.

Nous avons veillé à interroger des élus de différents courants politiques ${ }^{9}$ pour voir si les positions contradictoires qu'ils affichent ne recouvrent pas tout simplement les lignes de partage classiques entre partis et idéologies. Il en ressort que la totalité des élus de l'Ump considère les regroupements résidentiels de minorités ethniques comme un enjeu pour l'action publique, central dans leur acception de la mixité " sociale ». Cette position homogène des élus locaux de l'Ump contraste

\footnotetext{
7. Il s'agit d'Argenteuil, Aubervilliers, Bagneux, Bourg-la-Reine, Clichy-sous-Bois, Créteil, Fontenay-aux-Roses, Montfermeil et Sceaux. De décembre 2006 à mai 2007, nous y avons interrogé 132 personnes, élus municipaux, techniciens de la rénovation urbaine et de la politique de la ville et associations d'habitants. Cf. T. Kirszbaum, Les élus, la République et la mixité. Variations discursives et mise en débat de la norme nationale de mixité dans neuf communes franciliennes, PUCA, 2007.

8. On trouvera dans notre rapport de recherche de nombreux propos d'acteurs illustrant ces différentes positions.

9. Soit 10 Ump, 5 Udf, 11 Ps, 7 Pcf, 4 Verts et 2 associatifs (l'un classé à droite, l'autre à gauche).
} 
avec les clivages et hésitations qui traversent les autres courants politiques, les élus communistes paraissant les plus hésitants, oscillant entre une lecture ethnicisante et une lecture classiste, plus conforme à l'idéologie traditionnelle de leur parti.

Sans surprise, les mairies Ump sont aussi les plus enclines à interpréter la rénovation urbaine comme une action volontariste sur la composition ethno-raciale des quartiers ${ }^{10}$. Les mairies de gauche sont loin de manifester la même adhésion à cet objectif. Mais on peut identifier différentes cultures politiques qui rendent ces municipalités de gauche plus ou moins perméables à cette idée que le succès de la rénovation urbaine doit être apprécié à l'aune du "retour des Français de souche ". Les mairies socialistes de Clichy-sous-Bois et Créteil sont les plus sceptiques vis-à-vis d'une telle stratégie, mais les socialistes de Clichy-sousBois sont eux-mêmes écartelés entre deux courants. Le premier s'autoqualifie de "moderne ", prenant acte du caractère fortement ethnicisé de la ville, même s'il n'en tire pas toutes les conséquences pratiques, car il doit composer avec l'autre composante de la majorité municipale, celle des " anciens ", plus sensibles à la xénophobie de l'électorat blanc dans une commune où le Front national incarne la seule opposition véritable. Si la greffe du multiculturalisme a du mal à prendre à Clichysous-Bois, elle est moins éloignée de l'identité du socialisme de Créteil, héritier de la culture des villes nouvelles. Son maire, confortablement réélu depuis 1983, est issu de la mouvance du Psu et de la Cfdt, et l'influence de l'extrême-droite est beaucoup plus réduite dans sa ville qu’à Clichy. Aucun rejet des minorités ethniques n'est repérable dans la version cristollienne du socialisme municipal, mais les projets en cours de rénovation urbaine ne portent nulle trace de l'héritage multiculturaliste de la ville.

Les deux villes communistes de notre échantillon, Bagneux et Aubervilliers, incarnent elles aussi deux figures du communisme municipal. Bagneux se situe dans l'orthodoxie communiste, mais son combat pour la défense du logement social atteint ses limites dans le

10. Six villes de notre échantillon sont concernées par des opérations de rénovation urbaine. $\mathrm{Au}$ moment de l'enquête, deux étaient détenues par l'Ump (Argenteuil et Montfermeil), deux par le Ps (Clichy-sous-Bois et Créteil) et deux par le Pcf (Bagneux et Aubervilliers); Argenteuil et Aubervilliers sont passées depuis lors aux mains du Ps. 
contexte de valorisation rapide du sud des Hauts-de-Seine. Après une âpre discussion avec l'Agence nationale pour la rénovation urbaine (Anru), il a été décidé de démolir l'une des deux grandes barres Hlm du quartier des Blagis. Ce choix a été justifié par des raisons techniques et financières, mais la plupart des acteurs interrogés estiment que la présence de grandes familles d'origine africaine (que la municipalité s'efforce de disséminer dans différents quartiers de la ville) en a été la raison véritable. Le communisme d'Aubervilliers reste marqué quant à lui par la figure de son ancien maire Jack Ralite, incarnation de l'aile « libérale » du Pcf dont l'identité spécifique est mise à mal par la montée en puissance de l'allié/concurrent socialiste qui s'est finalement emparé de la mairie en mars 2008. Dans cette ville très spécialisée sur le plan social et ethnique, les projets de rénovation urbaine sont bien acceptés par l'ensemble de la gauche locale qui affiche un pragmatisme à toute épreuve, encourageant la gentrification du territoire (c'est-à-dire l'arrivée de ménages plus aisés venant de Paris), à laquelle résiste seule la tendance "rétro " du Pcf local, au nom de l'identité "ouvrière " de la ville. Les conséquences prévisibles de ces projets en termes de dispersion des ménages les plus précaires, là aussi souvent d'origine africaine, n'ont pas suscité beaucoup de réticence au sein de la majorité municipale : tous les courants politiques locaux portent d'une façon ou l'autre le discours du « ras-le-bol » concernant la fonction d'accueil de l'immigration qui incombe de facto à la ville d'Aubervilliers.

\section{D'autres stratēgies sont possibles}

Par-delà la diversité de leurs cultures politiques et en dépit de la variété des contextes sociaux et urbains, les stratégies locales de mixité apparaissent très peu différenciées dans les villes ayant engagé des projets de rénovation urbaine. Le diagnostic est partout le même : les quartiers "sensibles" sont handicapés et la population présente constitue leur handicap majeur. Partout, la mixité sociale constitue une référence centrale des projets de rénovation, et recouvre un sens univoque: substituer une population à une autre dans le but tacite de favoriser le « retour des Blancs ». 
Aucun des sites étudiés n'a sérieusement considéré d'autre option pour atteindre l'objectif de mixité sociale : ni celle d'une mixité " endogène " visant à élever la condition économique des habitants actuels des quartiers pour former en leur sein une classe moyenne qui deviendrait dominante et ne déménagerait pas, ni celle de la mixité dans les espaces non-résidentiels - comme l'école ou l'entreprise - par des actions résolument tournées vers la mobilité individuelle. Ces conceptions alternatives de la mixité sociale ont souvent les faveurs des techniciens du champ urbain ou social de la politique de la ville. Ceux que nous avons interrogés se montrent très critiques à l'égard de l'approche " exogène » de la mixité sociale, visant l'introduction artificielle de nouvelles catégories de population dans les quartiers. Beaucoup récusent la définition ethnique de la mixité sociale, dominante chez les élus et très répandue chez les bailleurs sociaux. Mais c'est dans leur for intérieur qu'ils formulent ces réserves : les stratégies alternatives ne sont jamais réellement discutées dans un cadre collectif, et les enjeux de l'ethnicité sont seulement évoqués dans un langage codé.

De même, aucune de ces villes n’a engagé de débat de fond avec les habitants sur la pertinence de la stratégie de mixité engagée au titre de la rénovation urbaine. Cette lacune tient pour partie aux contraintes institutionnelles d'élaboration des projets financés par l'Anru : délais, incertitudes, mode d'instruction hypertechnocratique des dossiers. Mais elle renvoie plus fondamentalement à l'impossibilité pour les élus d'associer les habitants à la conception des projets quand ces mêmes habitants font partie du problème à résoudre!

Dans tous les quartiers en rénovation urbaine, nous avons observé une ligne de fracture importante entre le groupe vieillissant des "anciens" habitants, de souche européenne, et celui des "nouveaux », pour beaucoup issus d'une immigration extra-européenne. Les " anciens", qui dominent les associations de locataires, sont aussi les participants les plus actifs aux diverses formes de " consultation-information» mises sur pied par les municipalités. Il est délicat pour les élus d'évoquer avec ces habitants l'objectif de mixité sociale, pourtant névralgique dans leurs projets urbains, car cet enjeu est souvent retraduit comme la volonté d'évincer les groupes les plus stigmatisés. Les populations originaires d'Afrique subsaharienne sont ici les premières visées, reléguant au second plan la figure du Maghrébin dans ce qui est 
décrit, par les tenants du "retour des Blancs », comme une impossible cohabitation résidentielle.

La position des militants associatifs appartenant aux minorités ethniques est elle-même ambivalente. Certains paraissent souffrir du regard porté par l'extérieur sur leur cité. Ils verraient d'un œil favorable l'arrivée de "Français de souche ", même si ce n'est pas leur priorité et pourvu que le droit de demeurer dans les futurs quartiers rénovés soit garanti pour tous, ce qui est loin d'être le cas. D'autres estiment que la mixité est d'ores et déjà effective dans ces quartiers, si l'on en juge par la diversité des origines ethniques représentées. Ils ne voient tout simplement pas où se trouve le ghetto.

Le contraste est alors saisissant avec les justifications institutionnelles de la rénovation urbaine. Si l'action publique ne sort pas indemne de l'ethnicisation croissante des rapports sociaux, on voit qu'elle est susceptible de l'alimenter, en véhiculant cette idée que les territoires où les minorités sont majoritaires sont illégitimes en toute circonstance. Cette focalisation obsessionnelle des pouvoirs publics français et de leurs relais locaux sur les regroupements résidentiels de minorités ethniques paraît bien être une singularité nationale. Dans beaucoup d'autres pays, les territoires concernés par rénovation urbaine ne sont pas forcément considérés comme des "ghettos" à casser, inéluctablement préjudiciables à l'intégration des minorités ethniques ${ }^{11}$. Plus que dans tout autre pays, le statut de la mixité a acquis un statut problématique dans la politique française de la ville qui y a perdu en chemin la notion même de pluralité d'approche des questions d'intégration dans la ville. Ouverte à l'origine à une pluralité d'orientations, la mixité " sociale » à l'échelle des quartiers de minorités a fini par devenir son seul horizon indiscutable, marginalisant d'autres options pourtant envisagées par le passé : la reconnaissance du caractère populaire et ethnique des quartiers et l'accès équitable aux ressources de la ville ${ }^{12}$.

Thomas Kirszbaum

11. J.-C. Driant et al., La mixité en Europe, une référence partagée, Créteil, Puca, 2006. Plus généralement, voir T. Kirszbaum, La mixité sociale dans l'habitat. Revue de la littérature dans une perspective comparative, Halde, La Documentation française, 2008.

12. T. Kirszbaum et R. Epstein, "Après les émeutes, comment débattre de la politique de la ville?", Regards sur l'actualité, 319, mars 2006. 